\title{
STARLIKE HYPERGEOMETRIC FUNCTIONS
}

E. P. MERKES AND W. T. SCOTT ${ }^{1}$

Let $a, b, c$ be complex numbers with $c \neq 0,-1,-2, \cdots$. For the hypergometric function $F(a, b, c ; z)$, which is defined in $|z|<1$ by the series

$$
1+\frac{a b}{c 1 !} z+\frac{a(a+1) b(b+1)}{c(c+1) 2 !} z^{2}+\cdots,
$$

the identities

$$
\begin{aligned}
c F(a, b+1, c ; z)= & a F(a+1, b+1, c+1 ; z) \\
& +(c-a) F(a, b+1, c+1 ; z), \\
c F^{\prime}(a, b, c ; z)= & a b F(a+1, b+1, c+1 ; z)
\end{aligned}
$$

are readily established.

When in the identity

$c(1-z) F(a, b+1, c ; z)=c F(a, b, c ; z)-(c-a) z F(a, b+1, c+1 ; z)$,

which is due to Gauss $[1$, p. 130], $c F(a, b+1, c ; z)$ is replaced by the right member of (2) the identity

$$
\begin{aligned}
a(1-z) F(a+1, b+1, c+1 ; z) & \\
& =c F(a, b, c ; z)-(c-a) F(a, b+1, c+1 ; z)
\end{aligned}
$$

is obtained. By (3) this can be rewritten for $z \neq 1$ as

$$
z \frac{F^{\prime}(a, b, c ; z)}{F(a, b, c ; z)}=\frac{b z}{1-z}\left[1-\frac{c-a}{c} \frac{F(a, b+1, c+1 ; z)}{F(a, b, c ; z)}\right]
$$

which is instrumental in the proof of the following:

TheOREM. Let $0<a<c$ and $-1<b \leqq a$. Then the function $u(z)$ $=z F(a, b, c ; z)$ is univalent and starlike with respect to the origin for $|z|<\rho$, where

$$
\rho=\frac{1}{1-b} \text { when }-1<b<0
$$

Presented to the Society, September 2, 1960; received by the editors December 8, 1960.

1 This research was supported by the United States Air Force Office of Scientific Research under Contract AF 49(638)-888. 


$$
\begin{array}{lll}
\rho=1 & \text { when } & 0 \leqq b \leqq 2 ; \\
\rho=\frac{1}{b-1} \text { when } & b>2 .
\end{array}
$$

Proof. A sufficient condition for $u(z)$ to be univalent and starlike in $|z|<r$ is [4]

$$
\operatorname{Re} s(z) \geqq 0,
$$$$
|z|<r
$$

where

$$
s(z)=z \frac{u^{\prime}(z)}{u(z)}=z \frac{F^{\prime}(a, b, c ; z)}{F(a, b, c ; z)}+1 .
$$

A result of Paydon and Wall [2] (see also [5, p. 46]) shows that if $0<g_{n}<1(n=1,2, \cdots)$, then the function $f(z)$ defined in $|z|<1$ by the continued fraction

$$
f(z)=\frac{g_{1}}{1}-\frac{\left(1-g_{1}\right) g_{2} z}{1}-\frac{\left(1-g_{2}\right) g_{3} z}{1}-\cdots
$$

satisfies

$$
\left|f(z)-\frac{1}{2-g_{1}}\right| \leqq \frac{1-g_{1}}{2-g_{1}}, \quad|z|<1 .
$$

A result of Wall $[5$, p. 283] states that when $f(z)$ is given by (7),

$$
\text { (9) } \frac{1-f(z)}{1-z f(z)}=\frac{1-g_{1}}{1}-\frac{g_{1}\left(1-g_{2}\right) z}{1}-\frac{g_{2}\left(1-g_{3}\right) z}{1}-\cdots \text {, }
$$

where the right member of (9) is obtained from the right member of (7) by replacing $g_{n}$ by $1-g_{n}$. In this case inequality (8) becomes

$$
\left|\frac{1-f(z)}{1-z f(z)}-\frac{1}{1+g_{1}}\right| \leqq \frac{g_{1}}{1+g_{1}}, \quad|z|<1,
$$

which is equivalent to the inequality

$$
\left|f(z)-\frac{g_{1}(1-\bar{z})}{1+g_{1}-z-\bar{z}+\left(1-g_{1}\right) z \bar{z}}\right|
$$

$$
\leqq \frac{g_{1}|1-z|}{1+g_{1}-z-\bar{z}+\left(1-g_{1}\right) z \bar{z}}, \quad|z|<1 .
$$

For 


$$
g_{2 n+1}=\frac{c-a+n}{c+2 n}, \quad g_{2 n+2}=\frac{c-b+n}{c+2 n+1}, \quad(n=0,1,2, \cdots),
$$

the function $(c-a) F(a, b+1, c+1 ; z) / c F(a, b, c ; z)$ is identified with the function $f(z)$ of $(7)[5$, p. 339] and

$$
s(z)=1+\frac{b z}{1-z}[1-f(z)]
$$

The requirement $0<g_{n}<1$ is equivalent to the conditions $0<a<c$, $-1<b<c$, and the second condition can be replaced by $-1<b \leqq a$ since $F(a, b, c ; z)$ is symmetric in $a$ and $b$. Thus, under the hypotheses of the Theorem, it follows from (10) that

where

$$
|s(z)-C| \leqq R, \quad|z|<1,
$$

$$
\begin{aligned}
& C=1+\frac{b z(c-a \bar{z})}{2 c-a+c(z+\bar{z})+a z \bar{z}}, \\
& R=\frac{|b z|(c-a)}{2 c-a+c(z+\bar{z})+a z \bar{z}},
\end{aligned}
$$

and hence (5) will hold if the function

$$
H(\theta)=\operatorname{Re} C-R
$$

is non-negative for $z=\mathrm{re}^{i \theta}, r<1$.

When $-1<b<0$,

$$
H(\theta)=1+\frac{b r(c \cos \theta-a r+c-a)}{2 c-a-2 c r \cos \theta+a r^{2}},
$$

and a simple computation shows that the minimum of $H(\theta)$ occurs for $\theta=0$. The statement (i) is then easily obtained from the requirement

$$
H(0)=1+\frac{b r}{1-r} \geqq 0 .
$$

Similarly, when $b>0$, the minimum of

$$
H(\theta)=1+\frac{b r(c \cos \theta-a r-c+a)}{2 c-a-2 c r \cos \theta+a r^{2}}
$$

occurs when $\theta=\pi$ and the requirement

$$
H(0)=1-\frac{b r}{1+r} \geqq 0
$$


leads to the statements (ii) and (iii).

It is evident that the result (ii) is sharp since $u(z)$ is not regular at $z=1$ except in the case where $b=0$.

A necessary and sufficient condition for a nonconstant regular function $F(z)$ to be univalent in $|z|<\rho$ and to map $|z|<\rho$ onto a convex region is that $z F^{\prime}(z)$ be univalent and starlike with respect to the origin for $|z|<\rho[3$, p. 221]. Since

$$
c z F^{\prime}(a, b, c ; z)=a b z F(a+1, b+1, c+1 ; z)
$$

by (3), application of the Theorem yields the following:

COROLlaRY. The function $F(a, b, c ; z)$, where $-1<a<c,-2<b \leqq a$, $a \neq 0, b \neq 0, c \neq 0$, is univalent in $|z|<\rho$ and maps $|z|<\rho$ onto a convex region if

$$
\begin{array}{llrl}
\rho & =-1 / b & \text { when } & -2<b<-1, \\
\rho & =1 & \text { when } & -1 \leqq b \leqq 1, b \neq 0, \\
\rho & =1 / b & \text { when } & b>1 .
\end{array}
$$

\section{REFERENCES}

1. C. F. Gauss, Disquisitiones generales circa seriem infinitam, Ges. Werke vol. 3 (1866) pp. 123-163.

2. J. F. Paydon and H. S. Wall, The continued fraction as a sequence of linear transformations, Duke Math. J. vol. 9 (1942) pp. 360-372.

3. Zeev Nehari, Conformal mapping, New York, McGraw-Hill, 1952.

4. L. Spācēk, Contribution a la theorie des fonctions univalents, Casopis Pest. Mat. Fys. vol. 62 (1932) pp. 12-19. 1948.

5. H. S. Wall, Analytic theory of continued fractions, New York, Van Nostrand

\section{MARQUetTE UNIVERSITY AND} NORTHWESTERN UNIVERSITY 